\title{
Iterative Multisymbol Noncoherent Reception of Coded CPFSK
}

\author{
Matthew C. Valenti \\ West Virginia University \\ Morgantown, WV \\ mvalenti@csee.wvu.edu
}

\author{
Shi Cheng \\ Arraycomm LLC \\ San Jose, CA \\ shi.cheng@gmail.com
}

\author{
Don Torrieri \\ U.S. Army Research Laboratory \\ Adelphi, MD \\ dtorr@arl.army.mil
}

\begin{abstract}
A multisymbol noncoherent receiver suitable for coded continuous-phase frequency-shift keying is developed and analyzed. Unlike coherently detected signals, the modulation index of the waveform does not need to be rational with a small denominator, and the oscillator only needs to be stable for the duration of a small group of symbols. The receiver is analyzed in terms of its symmetric information rate, demonstrating its potential when a capacity-approaching code is used and enabling the joint optimization of code rate and modulation index under a bandwidth constraint. The information-theoretic results are corroborated by bit-error-rate curves generated using a standardized turbo code in conjunction with iterative demodulation and decoding. It is shown that, while more robust than a system with coherent reception, the proposed system offers superior energy efficiency compared with conventional singlesymbol noncoherent reception.
\end{abstract}

\section{INTRODUCTION}

Continuous-phase frequency-shift keying (CPFSK) is a type of full-response continuous-phase modulation (CPM) that is characterized by the use of rectangular phase-shaping functions. Like other forms of CPM, CPFSK has attractive spectral characteristics because the smooth phase transitions between adjacent symbols minimize out-of-band power. A key benefit of CPFSK is that it can be noncoherently detected, which can provide significant complexity savings and improved robustness relative to coherent detection. The complexity savings of noncoherent detection are due to the fact that it does not require a trellis. In contrast, coherent detection requires a trellis with $Q$ states, where $Q$ is the denominator of the modulation index $h=P / Q$. While $h$ for the coherent detector must be rational, the noncoherent detector can work for any value of $h$. Furthermore, unlike the coherent detector, the noncoherent detector does not require knowledge of the initial phase or the set of allowable phases, which permits more robust operation.

If the channel- or oscillator-induced phase shift is stable over several symbols, a block or multisymbol noncoherent detector can be used to exploit the phase continuity within each block [1]. In this paper, we evaluate multisymbol noncoherent detection from an information-theoretic perspective. In particular, we compute the symmetric information rate, which is the modulation-constrained channel capacity under the assumption of independent and uniformly distributed (i.u.d.) inputs. The symmetric information rate is then used to determine the minimum required $\mathcal{E}_{b} / N_{0}$ when there is a bandwidth constraint. For a particular modulation order $M$ and channel type (AWGN or Rayleigh fading), the minimum $\mathcal{E}_{b} / N_{0}$ is found by optimizing the symmetric information rate with respect to the modulation index $h$ and code rate $r$. As one would anticipate, the minimum required $\mathcal{E}_{b} / N_{0}$ decreases with increasing block size.

The symmetric information rate of coherent CPM was considered by Ganesan [2] and Padmanabham et al. [3]. In [4], the symmetric information rate was used to jointly optimize the modulation index $h$ and code rate $r$ of coherent systems under a bandwidth constraint and strategies for code design were given. In [5], the symmetric information rate of CPFSK was considered for single-symbol noncoherent reception and the modulation index and code rate were jointly optimized. In general, the loss due to using conventional noncoherent reception is rather large, yet coherent receivers are difficult to implement. The purpose of this paper is to bridge the gap between coherent and noncoherent reception, which may be considered as limiting cases of the multisymbol noncoherent receiver with block lengths of either infinity or unity, respectively.

The remainder of this paper is organized as follows. A model of CPFSK is given in Section II, and the multisymbol noncoherent receiver is derived in Section III. The symmetric information rate of the system is discussed in Section IV, and the system parameters are optimized in Section V with respect to the symmetric information rate. In Section VI, simulation results are given showing the performance of multisymbol noncoherent detection when a turbo code is used.

\section{SySTEM MODEL}

A set of $K$ data bits is passed through a channel encoder, which outputs a length- $N_{c}$ vector $\mathbf{q}$ of $M$-ary symbols. The code rate is $r=K / N_{c} \leq \log _{2} M$ information bits per symbol. Instead of matching the alphabet of the encoder to that of the modulator, it is advantageous to use bit-interleaved coded modulation (BICM), which helps to counteract fading [6]. With BICM, the data is first passed through a binary encoder, bitwise interleaved, and then mapped to $M$-ary symbols.

For each entry of $\mathbf{q}$, the complex envelope of the continuous-time modulated signal $x_{i}(t)$ is chosen as the $q_{i}^{t h}$ signal of the set $\left\{s_{k}(t), k=0,1, \cdots, M-1\right\}$, where

$$
s_{k}(t)=\frac{1}{\sqrt{T_{s}}} e^{\frac{j 2 \pi k h t}{T_{s}}}, \quad t \in\left[0, T_{s}\right),
$$

$h$ is the modulation index, and $T_{s}$ is the symbol duration. In order to produce a more compact bandwidth, an additional phase $\phi_{i}$ is applied so that the phase transition from symbol 
to symbol is continuous. This additional phase is accumulated as

$$
\phi_{i}=2 q_{i} h \pi+\phi_{i-1} .
$$

The signal is transmitted over a block-fading channel. During the interval $i T_{s} \leq t \leq(i+1) T_{s}$, the complex envelope of the received signal is

$$
y_{i}(t)=a_{\lfloor i / L\rfloor} e^{j \theta_{\lfloor i / L\rfloor}} e^{j \phi_{i}} \sqrt{\mathcal{E}_{s}} x_{i}(t)+n_{i}(t),
$$

where $n_{i}(t)$ is additive white Gaussian noise (AWGN) with noise spectral density $N_{0}, \mathcal{E}_{s}$ is the energy per symbol, $L$ is the channel block length, and $a_{k} e^{j \theta_{k}}$ is the complex fading coefficient of channel block $k=\left\lfloor\frac{i}{L}\right\rfloor$. Two types of block channels are considered, Rayleigh fading and an AWGN channel that allows for an unknown phase when noncoherent detection is used. In both channels, $\theta_{k}$ is uniformly distributed over the range $[0,2 \pi)$ and independent from one block to the next. For the AWGN channel $a_{k}=1, \forall k$. In the block Rayleigh fading channel, the $a_{k}$ 's are Rayleigh distributed with equal energy and are independent from one block to the next.

The front-end of the multisymbol noncoherent detector is a bank of $M$ pairs of matched filters, with one pair matched to the in-phase and quadrature components of each tone. The matched filters are sampled at the symbol rate, producing an $M \times 1$ vector for the $i^{t h}$ symbol period [4]

$$
\mathbf{y}_{i}=a_{\lfloor i / L\rfloor} e^{j \theta_{\lfloor i / L\rfloor}} e^{j \phi_{i}} \sqrt{\mathcal{E}_{s}} \mathbf{x}_{i}+\mathbf{n}_{i}
$$

where $\mathbf{x}_{i}$ is a signal vector and $\mathbf{n}_{i}$ is a Gaussian-noise vector. When the modulated symbol $q_{i}=\nu, \mathbf{x}_{i}$ is the $\nu^{\text {th }}$ column of an $M \times M$ matrix $\mathbf{K}$ with $(j, k)^{t h}$ element

$$
K_{j, k}=\frac{\sin (\pi(k-j) h)}{\pi(k-j) h} e^{j \pi(k-j) h} .
$$

Note that the main diagonal of $\mathbf{K}$ is all ones. When $h$ is an integer, the off-diagonal terms are zero and the modulation is orthogonal. On the other hand, when $h$ is not an integer, the off-diagonal terms are complex values representing the intertone correlation. The noise vector $\mathbf{n}_{i}$ has covariance matrix $\mathbf{R}=E\left[\mathbf{n} \mathbf{n}^{H}\right]=N_{0} \mathbf{K}$ and is colored when $h$ is not an integer.

Consider a single symbol and drop the subscript $i$. Combine the two phase terms in (4) into a single phase $\psi=\theta+\phi$ which represents the received phase at the start of the symbol period. The symbol vector then becomes

$$
\mathbf{y}=a e^{j \psi} \sqrt{\mathcal{E}_{s}} \mathbf{x}+\mathbf{n}
$$

Since $\mathbf{n}$ is Gaussian, the vector $\mathbf{y}$ given $\mathbf{x}, a \sqrt{\mathcal{E}_{s}}$, and $\psi$ is Gaussian distributed with mean $a e^{j \psi} \sqrt{\mathcal{E}_{s}} \mathbf{x}$ and covariance $\mathbf{R}$. Thus, the conditional joint probability density function (pdf) is

$$
\begin{aligned}
& p\left(\mathbf{y} \mid \mathbf{x}, a \sqrt{\mathcal{E}_{s}}, \psi\right) \\
= & \frac{1}{\pi^{M} \operatorname{det}(\mathbf{R})} \exp ^{-\left(\mathbf{y}-a e^{j \psi} \sqrt{\mathcal{E}_{s}} \mathbf{x}\right)^{H} \mathbf{R}^{-1}\left(\mathbf{y}-a e^{j \psi} \sqrt{\mathcal{E}_{s}} \mathbf{x}\right)}
\end{aligned}
$$

The exponent of the conditional pdf can be simplified as

$$
-\left(\mathbf{y}-a e^{j \psi} \sqrt{\mathcal{E}_{s}} \mathbf{x}\right)^{H} \mathbf{R}^{-1}\left(\mathbf{y}-a e^{j \psi} \sqrt{\mathcal{E}_{s}} \mathbf{x}\right)
$$

$$
\begin{aligned}
= & -\mathbf{y}^{H} \mathbf{R}^{-1} \mathbf{y}-a^{2} \mathcal{E}_{s} \mathbf{x}^{H} \mathbf{R}^{-1} \mathbf{x} \\
& +2 \operatorname{Re}\left(a e^{-j \psi} \sqrt{\mathcal{E}_{s}} \mathbf{x}^{H} \mathbf{R}^{-1} \mathbf{y}\right) .
\end{aligned}
$$

Therefore, given $\mathbf{x}=\mathbf{k}_{\nu}$, where $\mathbf{k}_{\nu}$ represents the $\nu^{\text {th }}$ column of $\mathbf{K}$, the exponent becomes

$$
-\frac{\mathbf{y}^{H} \mathbf{K}^{-1} \mathbf{y}+a^{2} \mathcal{E}_{s}}{N_{0}}+2 \frac{a \sqrt{\mathcal{E}_{s}}}{N_{0}} \operatorname{Re}\left(e^{-j \psi} y_{\nu}\right) .
$$

Using this as the exponent of the conditional pdf and discarding terms that are common to all hypotheses, the conditional likelihood for each postulated symbol $\nu=\{0, \ldots, M-1\}$ becomes

$$
p\left(\mathbf{y} \mid \mathbf{x}=\mathbf{k}_{\nu}, a \sqrt{\mathcal{E}_{s}}, \psi\right) \propto \exp \left(2 \frac{a \sqrt{\mathcal{E}_{s}}}{N_{0}} \operatorname{Re}\left(e^{-j \psi} y_{\nu}\right)\right) .
$$

\section{Multisymbol Noncoherent Detection}

When the channel block length $L$ is sufficiently long, a multisymbol noncoherent detector of block length $N$ may be used, provided that $N \leq L$. Let the block of modulated symbols be $\mathbf{x}_{0}^{N-1}$ and the block of received symbols be $\mathbf{y}_{0}^{N-1}$. Here, $\mathbf{x}_{0}^{N-1}$ represents the set of $\left\{\mathbf{x}_{0}, \cdots, \mathbf{x}_{N-1}\right\}$, and $\mathbf{y}_{0}^{N-1}$ is similarly defined. The multisymbol noncoherent detector computes the probability $p\left(\mathbf{y}_{0}^{N-1} \mid \mathbf{x}_{0}^{N-1}\right)$ for each of the $M^{N}$ possible $\mathbf{x}_{0}^{N-1}$. Given the received amplitude $a \sqrt{\mathcal{E}_{s}}$ of the block and the initial received phase $\psi_{0}$ at the start of the block, the conditional probability of the block can be represented by the chain rule as

$$
\begin{aligned}
& p\left(\mathbf{y}_{0}^{N-1} \mid \mathbf{x}_{0}^{N-1}, a \sqrt{\mathcal{E}_{s}}, \psi_{0}\right) \\
= & \prod_{i=0}^{N-1} p\left(\mathbf{y}_{i} \mid \mathbf{y}_{0}^{i-1}, \mathbf{x}_{0}^{N-1}, a \sqrt{\mathcal{E}_{s}}, \psi_{0}\right) \\
= & \prod_{i=0}^{N-1} p\left(\mathbf{y}_{i} \mid \mathbf{x}_{i}, a \sqrt{\mathcal{E}_{s}}, \psi_{i}\right)
\end{aligned}
$$

where the second equality comes directly from the properties of Markov chains, and $\psi_{i}$ is given by (2) with $\psi_{i}=\phi_{i}+\theta$, where $\theta$ is the channel phase for the block (assumed constant since $N \leq L)$.

From (10) and (9), the conditional likelihood given the postulated block of symbols $\mathbf{q}=\left[q_{0}, \ldots, q_{N-1}\right]$ is

$p\left(\mathbf{y}_{0}^{N-1} \mid \mathbf{q}, a \sqrt{\mathcal{E}_{s}}, \psi_{0}\right) \propto \exp \left(2 \frac{a \sqrt{\mathcal{E}_{s}}}{N_{0}} \operatorname{Re}\left\{e^{-j \psi_{0}} \mu(\mathbf{q})\right\}\right)$

where

$$
\mu(\mathbf{q})=\sum_{i=0}^{N-1} y_{q_{i}} \exp \left\{-2 h \pi \sum_{k=0}^{i-1} q_{k}\right\} .
$$

The multisymbol noncoherent detector assumes $\psi_{0}$ has a uniform distribution over $[0,2 \pi)$. Marginalizing $p\left(\mathbf{y}_{0}^{N-1} \mid \mathbf{q}, a \sqrt{\mathcal{E}_{s}}, \psi_{0}\right)$ with respect to $\psi_{0}$ yields

$$
p\left(\mathbf{y}_{0}^{N-1} \mid \mathbf{q}, a \sqrt{\mathcal{E}_{s}}\right) \quad \propto \quad I_{0}\left(2 \frac{a \sqrt{\mathcal{E}_{s}}}{N_{0}}|\mu(\mathbf{q})|\right)
$$

where $I_{0}(\cdot)$ is the $0^{\text {th }}$ order modified Bessel function of the first kind. 
The multisymbol detector works as follows. For each hypothesis $\mathbf{q}$ of the $M^{N}$ hypotheses, it computes the conditional likelihood using (12) or its logarithm. A hard-decision detector merely takes the hypothesis that maximizes the likelihood. If a code matched to the modulation alphabet is used, then the likelihoods are passed directly into the decoder, which must be designed to accept such likelihoods. Alternatively, if BICM is used, then the demodulator computes the log-likelihood ratio for each code bit. Let $\mathbf{b}(\mathbf{q})=\left[b_{0}, \ldots, b_{\left(N \log _{2} M-1\right)}\right]$ be the set of $N \log _{2} M$ code bits associated with hypothetical code symbol vector $\mathbf{q}$. The LLR of the $k^{t h}$ code bit is

$$
\begin{array}{r}
\Lambda\left(b_{k}\right)=\log \frac{\sum_{\mathbf{q}: b_{k}(\mathbf{q})=1} p\left(\mathbf{y}_{0}^{N-1} \mid \mathbf{q}, a \sqrt{\mathcal{E}_{s}}\right) P\left[\mathbf{b}(\mathbf{q}) \backslash b_{k}(\mathbf{q})\right]}{\sum_{\mathbf{q}: b_{k}(\mathbf{q})=0} p\left(\mathbf{y}_{0}^{N-1} \mid \mathbf{q}, a \sqrt{\mathcal{E}_{s}}\right) P\left[\mathbf{b}(\mathbf{q}) \backslash b_{k}(\mathbf{q})\right]} \\
=\log \frac{\sum_{\mathbf{q}: b_{k}(\mathbf{q})=1} I_{0}\left(2 \frac{a \sqrt{\mathcal{E}_{s}}}{N_{0}}|\mu(\mathbf{q})|\right) P\left[\mathbf{b}(\mathbf{q}) \backslash b_{k}(\mathbf{q})\right]}{\sum_{\mathbf{q}: b_{k}(\mathbf{q})=0} I_{0}\left(2 \frac{a \sqrt{\mathcal{E}_{s}}}{N_{0}}|\mu(\mathbf{q})|\right) P\left[\mathbf{b}(\mathbf{q}) \backslash b_{k}(\mathbf{q})\right]},
\end{array}
$$

where the function $b_{k}(\mathbf{q})$ returns the $k^{t h}$ entry of the vector $\mathbf{b}(\mathbf{q})$ and $P\left[\mathbf{b}(\mathbf{q}) \backslash b_{k}(\mathbf{q})\right]$ is the a priori probability of $\mathbf{b}(\mathbf{q})$ excluding bit $b_{k}(\mathbf{q})$. In a non-iterative BICM receiver, the $a$ priori probabilities are all equal and therefore may be dropped from the logarithm. In an iterative BICM receiver (c.f. BICMID [7]), then the a priori probabilities may be determined from the extrinsic information fed back from the decoder. Note that BICM-ID is only advantageous when the number of hypothesis is nonbinary, i.e. when $M^{N}>2$.

\section{Symmetric Information RATE}

The capacity of a channel with input block $\mathbf{X}=\mathbf{x}_{0}^{N-1}$ and output block $\mathbf{Y}=\mathbf{y}_{0}^{N-1}$ is

$$
C=\max _{p(\mathbf{X})} I(\mathbf{X} ; \mathbf{Y}),
$$

where $p(\mathbf{X})$ is the joint pdf of $\mathbf{X}$, the average mutual information is

$$
I(\mathbf{X} ; \mathbf{Y})=E[i(\mathbf{X} ; \mathbf{Y})]
$$

and

$$
i(\mathbf{X} ; \mathbf{Y})=\log \frac{p(\mathbf{X}, \mathbf{Y})}{p(\mathbf{X}) p(\mathbf{Y})}=\log \frac{p(\mathbf{Y} \mid \mathbf{X})}{p(\mathbf{Y})}
$$

Note that the expectation in (15) is with respect to the joint pdf $p(\mathbf{X}, \mathbf{Y})$. When a base-2 logarithm is used, then (14) has units of bits per block.

The capacity given in (14) is found by optimizing with respect to the distribution of the input blocks $\mathbf{X}$. This optimization may result in a nonuniform distribution. However, most practical systems assume a uniform distribution of channel inputs, and therefore the inputs are constrained to be independent and uniformly distributed (i.u.d.). The capacity under the constraint of i.u.d. inputs is called the symmetric information rate, and is found by dropping the maximization in (14) and assuming i.u.d. inputs when computing the average mutual information. For brevity, we also refer to the symmetric information rate as the i.u.d. capacity or just capacity in this paper.

Assume equiprobable $\mathbf{X}, p(\mathbf{X})=1 / M^{N}$, and let $\mathcal{S}$ be the set of $M^{N}$ possible values of $\mathbf{X}$. Because $p(\mathbf{Y})=$ $\sum_{\mathbf{X}^{\prime} \in \mathcal{S}} p\left(\mathbf{Y} \mid \mathbf{X}^{\prime}\right) p\left(\mathbf{X}^{\prime}\right)=M^{-N} \sum_{\mathbf{X}^{\prime} \in \mathcal{S}} p\left(\mathbf{Y} \mid \mathbf{X}^{\prime}\right)$, we may rewrite (16) as

$$
i(\mathbf{X} ; \mathbf{Y})=N \log M+\log \frac{p(\mathbf{Y} \mid \mathbf{X})}{\sum_{\mathbf{X}^{\prime} \in \mathcal{S}} p\left(\mathbf{Y} \mid \mathbf{X}^{\prime}\right)} .
$$

Substituting the conditional pdf of the multisymbol noncoherent detector given by (12) into (17) and (15) results in a symmetric information rate

$$
I(\mathbf{X} ; \mathbf{Y})=N \log M+E\left[\log \frac{I_{0}\left(\frac{2 a \sqrt{\mathcal{E}_{s}}|\mu(\mathbf{q})|}{N_{0}}\right)}{\sum_{\mathbf{q}^{\prime} \in \mathcal{Q}} I_{0}\left(\frac{2 a \sqrt{\mathcal{E}_{s}}\left|\mu\left(\mathbf{q}^{\prime}\right)\right|}{N_{0}}\right)}\right]
$$

where $\mathcal{Q}$ is the set of $M^{N}$ possible values of $\mathbf{q}$ and the expectation is taken over the ensemble of all possible transmitted $\mathbf{q}$ and received $\mathbf{y}_{0}^{N-1}$. The above expectation can be found using Monte Carlo integration. To express (18) in terms of bits per channel symbol, use a base- 2 logarithm and divide the result by $N$, i.e. the number of symbols per block.

As an example, Fig. 1 shows the i.u.d. capacity (in bits per channel symbol) of multisymbol noncoherent detection of minimum-shift keying (MSK), i.e. $h=0.5$ and $M=2$, for several different block sizes in AWGN. The rightmost curve $(N=1)$ is the capacity of single-symbol noncoherent detection, while the leftmost curve is the coherent capacity [4]. By increasing $N$ from 1 to 4 , the gain at code-rate 0.5 is about $5 \mathrm{~dB}$, and it is only $3.5 \mathrm{~dB}$ worse than coherent detection. As $N$ increases, the capacity continues to approach that of coherent detection. As $N \rightarrow \infty$, we conjecture that the multisymbol noncoherent capacity converges to the coherent capacity since the two receivers become identical (except for knowledge of the initial phase $\phi_{0}$, which becomes irrelevant for large $N$ ).

\section{BANDWIDTh-CONSTRAined PARAMETER OPTIMIZATION}

The bandwidth of coded CPFSK is a function of the modulation index, code rate, and modulation order. When the bandwidth is constrained to not exceed $B_{\max }$, there is a particular combination of these parameters that minimizes the value of $\mathcal{E}_{b} / N_{0}$ required to achieve an arbitrarily low bit error rate. While one could search for the optimal combination of parameters by exhaustively simulating every possible choice of parameters with an actual capacity-approaching code, a more efficient way to search would be to leverage the capacity analysis discussed in the previous section. The benefit of using capacity analysis is that it provides a fairly accurate prediction 


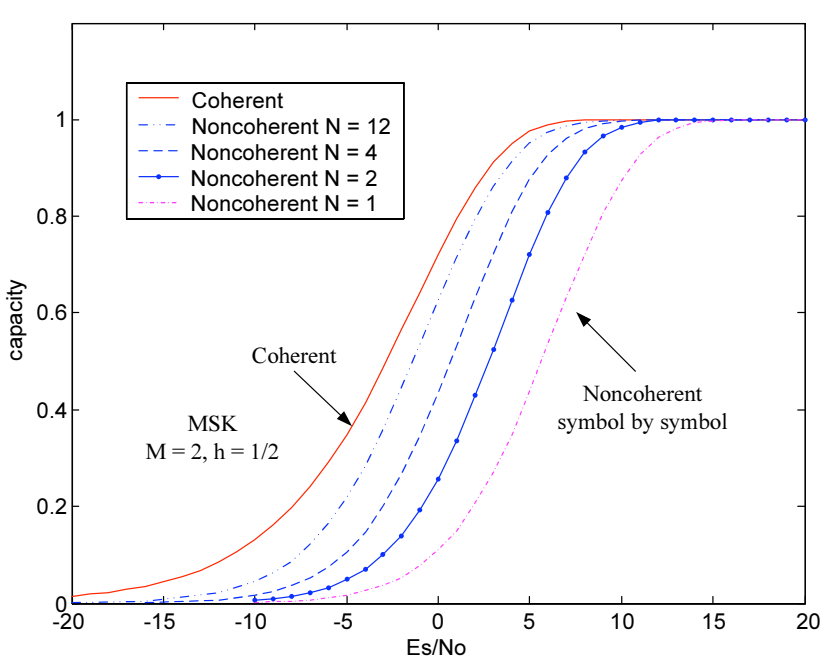

Fig. 1. Symmetric information rate of MSK over an AWGN channel with either $N$-symbol noncoherent detection or coherent detection.

of the performance that can be achieved with a capacityapproaching code without requiring that the code actually be simulated. Of course, once a good design is identified, it should be simulated using the proposed receiver and an actual code such as a turbo code, thereby confirming the effectiveness of the design.

In order to optimize under a bandwidth constraint, the influence of the system parameters upon bandwidth must be quantified. First consider an uncoded signal $s(t)$ with power spectral density (PSD) $\Phi_{s}(f)$. Given the PSD, the $99 \%$ power bandwidth of the uncoded $s(t)$ is defined as the value of $B_{99}$ that satisfies

$$
\int_{-B_{99} / 2}^{B_{99} / 2} \Phi_{s}(f) d f=0.99 \int_{-\infty}^{\infty} \Phi_{s}(f) d f .
$$

The PSD $\Phi_{s}(f)$ of CPFSK is well known and can be found in texts such as Section 3.4-5 of [8]. When channel coding is used, the required bandwidth increases as the code rate decreases. Define the normalized bandwidth of the coded signal to be

$$
\beta=\frac{B_{99}}{R_{b}},
$$

where $R_{b}=r / T_{s}$ is the data rate in bits per second and $\beta$ is in units of $\mathrm{Hz} / \mathrm{bps}$.

Assume that the normalized bandwidth must not exceed a value $\beta_{\max }$. The goal of the capacity-based optimization is to minimize the $\mathcal{E}_{b} / N_{0}$ required for reliable communication under this bandwidth constraint, which is achieved by jointly optimizing the parameters $h$ and $r$ for the given value of $M$, channel type (AWGN or fading), and decoder type (e.g., the block length $N$ used by the multisymbol decoder). To perform the optimization, start with an arbitrary value of $h$. For this value of $h$, there will be a minimum permissible code rate $r_{\text {min }}$. Using a code rate lower than $r_{\text {min }}$ with this $h$ will violate the bandwidth constraint. Next, the symmetric information rate is computed from (18) and used to determine the value of $\mathcal{E}_{s} / N_{0}$ required to achieve $I(\mathbf{X} ; \mathbf{Y})=r_{\text {min }}$, and the corresponding $\mathcal{E}_{b} / N_{o}=\left(\mathcal{E}_{s} / N_{o}\right) / r_{\text {min }}$ is determined. This will normally be the minimum $\mathcal{E}_{b} / N_{o}$ required for this value of $h$. However, due to the noncoherent-combining penalty, it is possible that a rate higher than $r_{\min }$ will provide a lower required $\mathcal{E}_{b} / N_{o}$ despite having a normalized bandwidth that is actually less than $\beta_{\max }$. Thus, in order to account for this possibility, all rates $r \in\left[r_{\text {min }}, \log _{2} M\right]$ must be considered when searching for the minimum $\mathcal{E}_{b} / N_{o}$. This step is especially important when the bandwidth constraint is loose.

The above procedure will give the minimum $\mathcal{E}_{b} / N_{o}$ and the optimum code rate for each valid value of $h$ that is considered. In order to determine the global minimum, then the process must be repeated for all $h$. In practice, it is sufficient to consider closely sampled values of $h$. Note that there is an upper limit on $h$ such that even if a rate $r=\log _{2} M$ code were used, the bandwidth constraint would be violated.

Fig. 2 shows results under bandwidth constraint $\beta_{\max }=2$ $\mathrm{Hz} / \mathrm{bps}$ in AWGN with $M=2$ tones. Multisymbol reception with $N=1,2$ and 4 was considered. In the simulations used to compute the symmetric information rate, the channel block length is set to $L=N$. While a larger value of $L$ could have been selected, the symmetric information rate does not depend on the value of $L$ provided that $L \geq N$ and a sufficiently large number of trials is performed. For each value of $N$, the minimum $\mathcal{E}_{b} / N_{0}$ was determined for values of $h$ that are multiples of 0.01 and within a valid range $0.01 \leq h \leq 0.86$. As a point of comparison, the capacity for coherent CPFSK is shown for specific values of $h$, namely those $h=P / Q$ with integer $Q$ satisfying $2 \leq Q \leq 5$. The values for coherent reception are from [4] . For each type of receiver, the required $\mathcal{E}_{b} / N_{0}$ is minimized for $h=0.6$. The corresponding code rate for this value of $h$ is $r=0.64$ (the bandwidth constraint is too tight for the noncoherent-combining penalty to require an $r$ greater than this minimum). For conventional single-symbol noncoherent detection $(N=1)$, the system requires $\mathcal{E}_{b} / N_{0}=8.08 \mathrm{~dB}$ while for coherent detection the system requires $\mathcal{E}_{b} / N_{0}=-0.10 \mathrm{~dB}$. This gap is bridged by multisymbol noncoherent reception. For $N=2$, the required $\mathcal{E}_{b} / N_{0}=5.15 \mathrm{~dB}$ while for $N=4$ the required $\mathcal{E}_{b} / N_{0}=3.67$ $\mathrm{dB}$. Thus, these capacity results predict that using length $N=4$ blocks will provide a gain of over $4.4 \mathrm{~dB}$ relative to single-symbol noncoherent detection in an AWGN channel. However, for $M=2$, there is still a loss of about $3.7 \mathrm{~dB}$ when using 4-block multisymbol noncoherent detection instead of coherent detection.

Fig. 3 shows the performance of multisymbol noncoherent reception in Rayleigh fading with $\beta_{\max }=2 \mathrm{~Hz} / \mathrm{bps}, M=2$, and $N=\{1,2,4\}$. The performance of coherent reception is not shown because it is not given in [4] and it is generally not feasible for the coherent receiver to track the phase of a block fading channel when the blocks are so short. For this bandwidth constraint, the value of $h$ that minimizes the required $\mathcal{E}_{b} / N_{0}$ is again $h=0.6$, and the corresponding code rate is $r=0.64$. The values for the minimum $\mathcal{E}_{b} / N_{0}$ are 10.78 


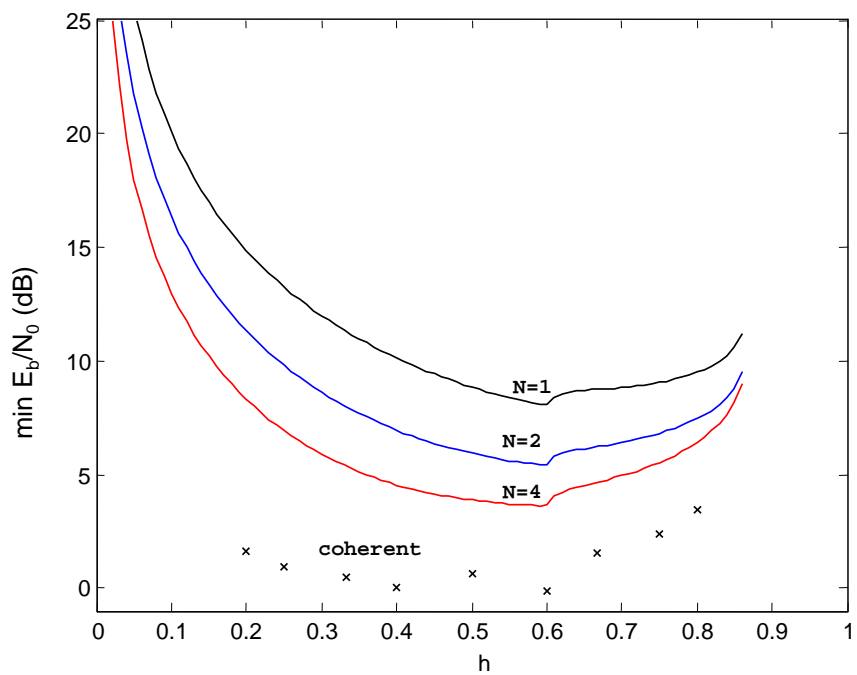

Fig. 2. Minimum $\mathcal{E}_{b} / N_{0}$ required for binary CPFSK to achieve an arbitrarily low error rate versus modulation index $h$ in AWGN with $M=2$ under bandwidth constraint $\beta_{\max }=2 \mathrm{~Hz} / \mathrm{bps}$ using $N$-symbol noncoherent detection with $N=\{1,2,4\}$. As a point of comparison, the minimum $\mathcal{E}_{b} / N_{0}$ required for coherent CPFSK is also shown [4]

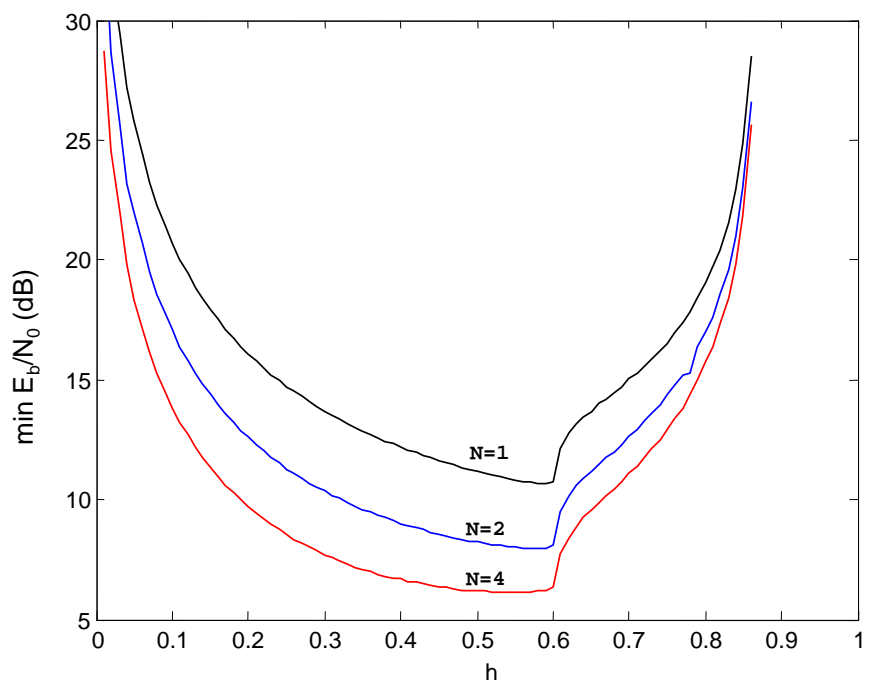

Fig. 3. Minimum $\mathcal{E}_{b} / N_{0}$ required for binary CPFSK to achieve an arbitrarily low error rate versus modulation index $h$ in Rayleigh fading with $M=2$ under bandwidth constraint $\beta_{\max }=2 \mathrm{~Hz} / \mathrm{bps}$ using $N$-symbol noncoherent detection with $N=\{1,2,4\}$.

$\mathrm{dB}, 8.10 \mathrm{~dB}$, and $6.31 \mathrm{~dB}$ for $N=1,2$, and 4 , respectively. As with the AWGN channel, there is a gain of more than $4.4 \mathrm{~dB}$ when length $N=4$ blocks are used instead of conventional single-symbol detection.

Results were also obtained for quaternary CPFSK $(M=4)$ and $\beta_{\max }=2$ and are shown in Fig. 4 for the AWGN channel and Fig. 5 for the Rayleigh fading channel. With coherent reception, the minimum $\mathcal{E}_{b} / N_{0}$ in AWGN for $M=4$ is -0.32 $\mathrm{dB}$. With multisymbol noncoherent reception, the values for the minimum $\mathcal{E}_{b} / N_{0}$ over the AWGN channel are $5.33 \mathrm{~dB}$, $3.51 \mathrm{~dB}$, and $2.20 \mathrm{~dB}$ for $N=1,2$, and 4 , respectively. Thus, the gain of $N=4$ multisymbol noncoherent reception in AWGN is about $3.1 \mathrm{~dB}$ relative to single-symbol noncoherent

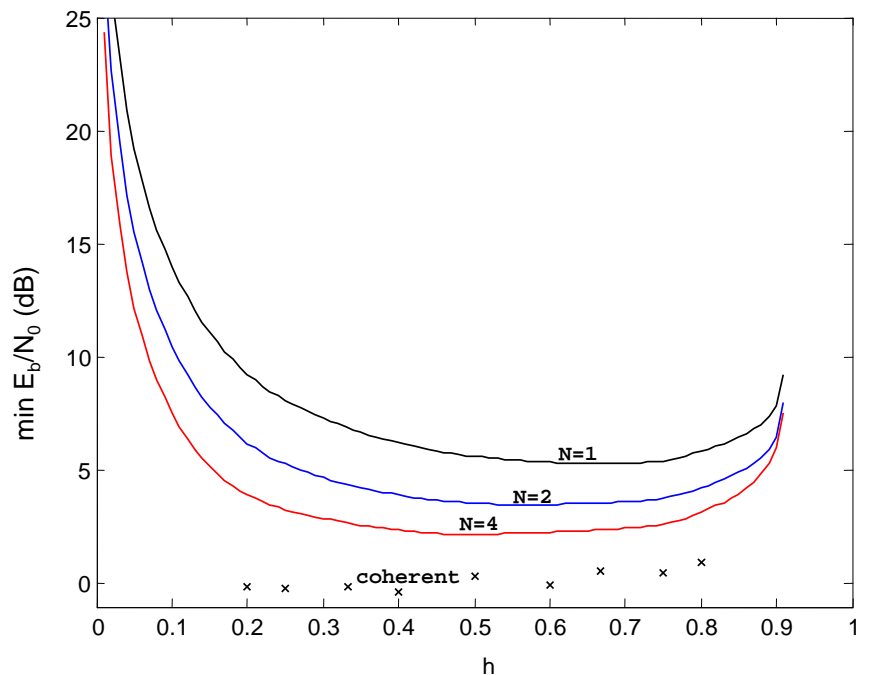

Fig. 4. Minimum $\mathcal{E}_{b} / N_{0}$ required for binary CPFSK to achieve an arbitrarily low error rate versus modulation index $h$ in AWGN with $M=4$ under bandwidth constraint $\beta_{\max }=2 \mathrm{~Hz} / \mathrm{bps}$ using $N$-symbol noncoherent detection with $N=\{1,2,4\}$. As a point of comparison, the minimum $\mathcal{E}_{b} / N_{0}$ required for coherent CPFSK is also shown [4]

reception, though $N=4$ multisymbol noncoherent reception has a loss of $2.5 \mathrm{~dB}$ relative to coherent reception. The gain achieved by increasing $M$ from 2 to 4 is only $0.22 \mathrm{~dB}$ for the coherent receiver, but for the noncoherent receiver it is 2.75 $\mathrm{dB}, 1.64 \mathrm{~dB}$, and $1.47 \mathrm{~dB}$ for $N=1,2$, and 4 , respectively. It is observed that the multisymbol noncoherent receiver is better able to exploit the larger signal set than does the coherent receiver.

In the Rayleigh fading channel with $M=4$, the values for the minimum $\mathcal{E}_{b} / N_{0}$ indicated by Fig. 5 are $8.17 \mathrm{~dB}$, $5.87 \mathrm{~dB}$, and $4.31 \mathrm{~dB}$ for $N=1,2$, and 4 , respectively. The gain of $N=4$ multisymbol noncoherent reception in the Rayleigh fading channel with $M=4$ is over $3.8 \mathrm{~dB}$ relative to single-symbol noncoherent reception. For the multisymbol noncoherent receiver, the gain from using $M=4$ instead of $M=2$ is $2.61 \mathrm{~dB}, 2.23 \mathrm{~dB}$, and $2.00 \mathrm{~dB}$ for $N=1,2$, and 4 , respectively, again demonstrating that the multisymbol noncoherent receiver is able to exploit the larger signal set.

\section{Turbo-Coded Performance}

Simulations were performed to demonstrate the achievable performance when an actual channel code is used, thereby confirming the validity of the information-theoretic bounds. Systems with CPFSK modulation and $M=2$ and $M=4$ were considered. For systems with $M=2$, the modulation index was set to $h=0.6$, which is the optimal value for the single-symbol noncoherent detector under bandwidth constraint $\beta_{\max }=2 \mathrm{~Hz} / \mathrm{bps}$ according to the informationtheoretic analysis given in the last section. The code rate was set to its corresponding optimal value $r=0.64$. Data was encoded using the turbo code specified by the UMTS standard [9], which was selected due to its widespread use and its ability to handle a variety of code rates. The rate $r=0.64$ was achieved by using a message length of $K=4800$ bits 


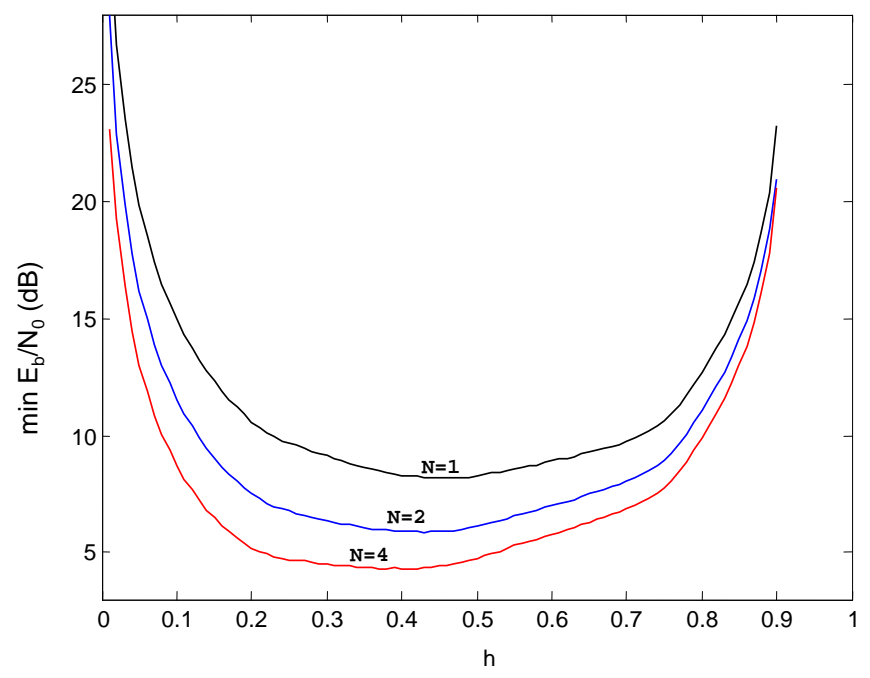

Fig. 5. Minimum $\mathcal{E}_{b} / N_{0}$ required for binary CPFSK to achieve an arbitrarily low error rate versus modulation index $h$ in Rayleigh fading with $M=4$ under bandwidth constraint $\beta_{\max }=2 \mathrm{~Hz} / \mathrm{bps}$ using $N$-symbol noncoherent detection with $N=\{1,2,4\}$.

and a codeword length of $N_{c}=7500$ bits. The demodulator was implemented using the proposed noncoherent $N$-symbol demodulator with $N=1,2$ and 4 . The turbo code was decoded using 30 iterations of the log-MAP algorithm [8]. When $M^{N}>2$, a BICM-ID receiver was used, in which case the soft-output from each decoder iteration was used by the demodulator as a priori information.

Simulations were run for both an AWGN channel and a Rayleigh fading channel. Fig. 6 shows results for the AWGN channel with $M=2$. Three curves are shown corresponding to length $N=1,2$, and 4 demodulator blocks. In addition, a vertical line is shown for each value of $N$ which corresponds to the minimum $\mathcal{E}_{b} / N_{0}$ found from the previously discussed information-theoretic analysis. The value of $\mathcal{E}_{b} / N_{0}$ required for the turbo-coded system to achieve a BER of $10^{-5}$ in AWGN is $8.90 \mathrm{~dB}, 6.13 \mathrm{~dB}$, and $4.44 \mathrm{~dB}$ for $N=1,2$, and 4 , respectively. These values are between 0.80 and $0.98 \mathrm{~dB}$ from the corresponding information-theoretic bounds, indicating that it is possible to design a system that is capable of coming within a decibel of the bounds.

Fig. 7 shows results for the Rayleigh fading channel with $M=2$. In the simulations, the fading amplitude was held constant for blocks of $L$ consecutive symbols and varied independently from one block to the next. In order to maximize the number of independent fades per codeword, we set the value of $L$ to the minimum value required by the multisymbol noncoherent detector, which is $N=L$. As in the previous figure, a vertical line is shown for each value of $N$ which corresponds to the minimum $\mathcal{E}_{b} / N_{0}$ found from the informationtheoretic analysis. The value of $\mathcal{E}_{b} / N_{0}$ required for the turbocoded system to achieve a BER of $10^{-5}$ in Rayleigh fading is $12.04 \mathrm{~dB}, 9.41 \mathrm{~dB}$, and $7.72 \mathrm{~dB}$ for $N=1,2$, and 4 , respectively. These values are between 1.26 and $1.41 \mathrm{~dB}$ from the corresponding information-theoretic bounds. While the gap between the theoretical $\mathcal{E}_{b} / N_{0}$ and the value required with the

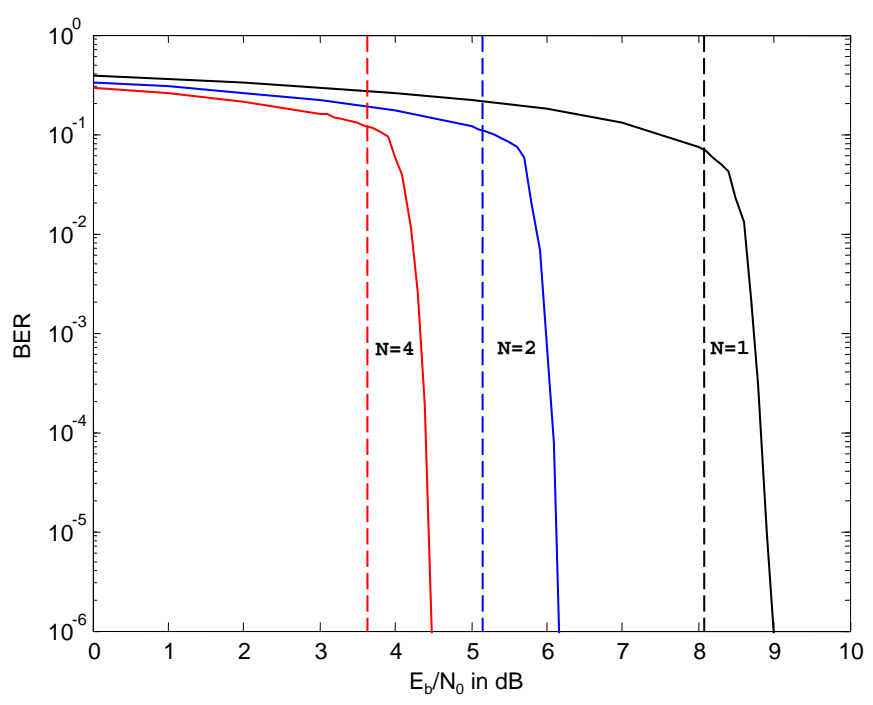

Fig. 6. BER vs. $\mathcal{E}_{b} / N_{0}$ in AWGN of a CPFSK system using $M=2, h=$ $0.6, r=0.64$ and $N$-symbol noncoherent detection with $N=\{1,2,4\}$.

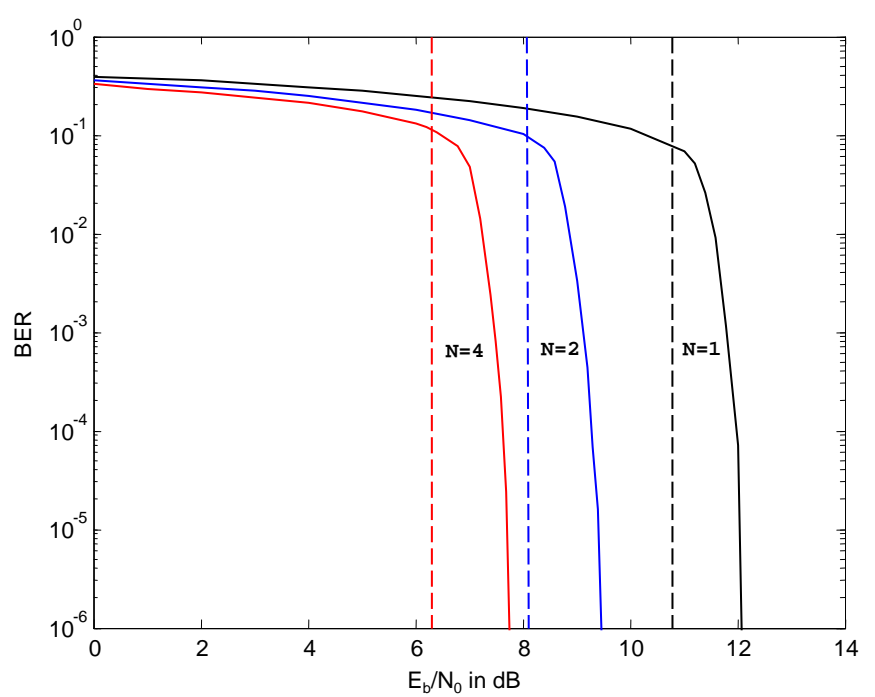

Fig. 7. BER vs. $\mathcal{E}_{b} / N_{0}$ in Rayleigh fading of a CPFSK system using $M=2, h=0.6, r=0.64$ and $N$-symbol noncoherent detection with $N=\{1,2,4\}$.

actual turbo code is slightly higher for Rayleigh fading than it is for AWGN, it is feasible to design a system that can come within $1.5 \mathrm{~dB}$ of the bounds in Rayleigh fading.

For systems with $M=4$, the modulation index was set to $h=0.67$ for the AWGN channel and $h=0.45$ for the Rayleigh fading channel. These are the information-theoretic optimal values for the single-symbol noncoherent detector under bandwidth constraint $\beta_{\max }=2 \mathrm{~Hz} / \mathrm{bps}$. The UMTS standard turbo code was again used, with the rate set to $r=5100 / 6528$ for the AWGN channel and $r=3800 / 6528$ for the Rayleigh fading channel. The codeword length was 6528 bits for both channels. For all values of $N, 30$ iterations of BICM-ID reception was performed by using a combination of the proposed receiver and the log-MAP decoding algorithm.

Fig. 8 shows results for the AWGN channel with $M=4$ 


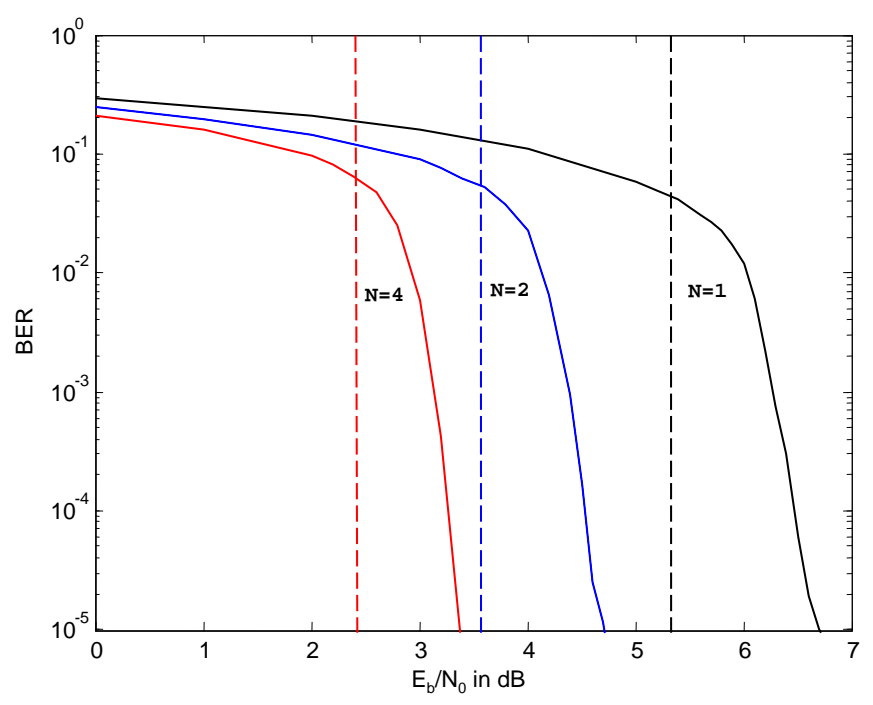

Fig. 8. BER vs. $\mathcal{E}_{b} / N_{0}$ in AWGN of a CPFSK system using $M=4, h=$ $0.67, r=0.78$ and $N$-symbol noncoherent detection with $N=\{1,2,4\}$.

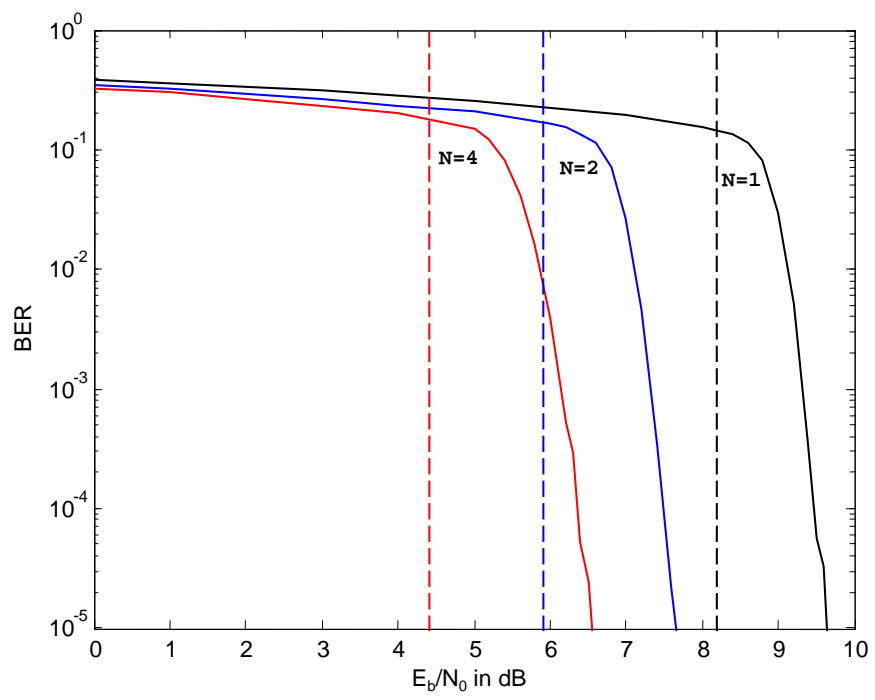

Fig. 9. BER vs. $\mathcal{E}_{b} / N_{0}$ in Rayleigh fading of a CPFSK system using $M=4, h=0.45, r=0.58$ and $N$-symbol noncoherent detection with $N=\{1,2,4\}$.

and three values of $N$. The value of $\mathcal{E}_{b} / N_{0}$ required for the turbo-coded system to achieve a BER of $10^{-5}$ in AWGN is $6.70 \mathrm{~dB}, 4.72 \mathrm{~dB}$, and $3.37 \mathrm{~dB}$ for $N=1,2$, and 4 , respectively. These values are between 0.94 and $1.37 \mathrm{~dB}$ from the corresponding information-theoretic bounds, which are indicated by the dashed lines.

Fig. 9 shows results for the Rayleigh fading channel with $M=4$. The value of $\mathcal{E}_{b} / N_{0}$ required for the turbo-coded system to achieve a BER of $10^{-5}$ in Rayleigh fading is $9.63 \mathrm{~dB}, 7.65 \mathrm{~dB}$, and $6.54 \mathrm{~dB}$ for $N=1,2$, and 4 , respectively. These values are between 1.44 and $2.13 \mathrm{~dB}$ from the corresponding information-theoretic bounds.

\section{CONCLUSION}

Multisymbol noncoherent demodulation is an attractive compromise between coherent demodulation, which is often difficult and complex to implement, and single-symbol noncoherent demodulation, which has poor energy efficiency. The performance of a multisymbol noncoherent system can be improved by using a capacity-approaching code, such as a turbo code. To fully exploit the benefits of the channel code, the demodulator developed in this paper uses soft-input, softoutput demodulation over blocks of multiple received symbols.

The performance of a system that uses a capacityapproaching code and the proposed demodulator may be predicted by using the symmetric information rate. The performance depends on several parameters, including the choice of modulation index $h$ and code rate $r$. When there is a bandwidth constraint, there is a combination of $h$ and $r$ that minimizes the $\mathcal{E}_{b} / N_{0}$ required for reliable signaling. The symmetric information rate can be used to identify the optimal values of these parameters for a particular bandwidth constraint, modulation order $M$, demodulator block length $N$, and channel type. When the optimal parameters are chosen and binary modulation is used, the 4-symbol demodulator outperforms the single-symbol demodulator by about 4.4-4.5 $\mathrm{dB}$ in both AWGN and Rayleigh fading channels, yet is still about $3.7 \mathrm{~dB}$ worse than the coherent demodulator.

Once the optimal parameters have been identified, a complete system can be designed by incorporating an outer errorcorrecting code. While the code could be optimized for the particular system, reasonable performance can be achieved by using an "off-the-shelf" standardized code. In particular, if the standardized UMTS turbo-code is used and $M=2$ tones, then performance within $1 \mathrm{~dB}$ of the information-theoretic bound can be achieved in AWGN and performance within $1.5 \mathrm{~dB}$ of the bound can be achieved over a Rayleigh fading channel. With $M=4$ tones are used with the UMTS turbo code, performance is within $1.4 \mathrm{~dB}$ of the information-theoretic bound in AWGN and within $2.2 \mathrm{~dB}$ of the bound in Rayleigh fading.

\section{REFERENCES}

[1] M. K. Simon and D. Divsalar, "Maximum-likelihood block detection of noncoherent continuous phase modulation," IEEE Trans. Commun., vol. 41, pp. 90-98, Jan. 1993.

[2] A. Ganesan, "Capacity estimation and code design principles for continuous phase modulation (CPM)," Master's thesis, Texas A\&M University, College Station, TX, May 2003.

[3] K. Padmanabhan, S. Ranganathan, S. P. Sundaravaradham, and O. M. Collins, "General CPM and its capacity," in Proc. IEEE Int. Symp. on Inform. Theory (ISIT), (Adelaide, Australia), pp. 750-754, Sept. 2005.

[4] S. Cheng, M. C. Valenti, and D. Torrieri, "Coherent continuous-phase frequency-shift keying: Parameter optimization and code design," IEEE Trans. Wireless Comm., vol. 8, pp. 1792-1802, April 2009.

[5] S. Cheng, R. I. Seshadri, M. Valenti, and D. Torrieri, "The capacity of noncoherent continuous-phase frequency shift keying," in Proc. Conference on Information Sciences and Systems (CISS), (Baltimore, MD), March 2007.

[6] G. Caire, G. Taricco, and E. Biglieri, "Bit-interleaved coded modulation," IEEE Trans. Inform. Theory, vol. 44, pp. 927-946, May 1998.

[7] X. Li and J. A. Ritcey, "Bit-interleaved coded modulation with iterative decoding," IEEE Commun. Letters, vol. 1, pp. 169-171, Nov. 1997.

[8] J. G. Proakis and M. Salehi, Digital Communications. New York, NY: McGraw-Hill, Inc., fifth ed., 2008.

[9] European Telecommunications Standards Institute, "Universal mobile telecommunications system (UMTS): Multiplexing and channel coding (FDD)," 3GPP TS 25.212 version 3.4.0, Sept. 232000. 